\title{
Treatment of laryngeal telangiectatic lesions in a patient diagnosed with hereditary haemorrhagic telangiectasia
}

\begin{abstract}
We here present a case concerning a 69year old female patient with Hereditary Haemorrhagic Telangiectasia (HHT). She was suffering from hoarseness due to a telangiectatic lesion on the right vocal cord. The lesion was treated with laser and the voice improved markedly, which is documented by phonetogram measurement. Patients with HHT who complain of hoarseness should be referred to an otolaryngologist for evaluation. If telangiectatic lesions are identified at the vocal cords, these may explain the hoarseness and should be treated with laser surgery, in order to improve the voice and prevent re-bleeding. The treatment can be performed without any harm to the vocal mucosa, and a marked improvement of the voice may be noted.
\end{abstract}

Keywords: hht, telangiectatic lesions, larynx
Volume 2 Issue 6 - 2015

\author{
Anette Drøhse Kjeldsen, Trine Printz, \\ Camilla Slot Mehlum, Ågot Møller Grøntved \\ Department of Otorhinolaryngology, Odense University \\ Hospital, Denmark
}

Correspondence: Anette Drøhse Kjeldsen, Department of Otorhinolaryngology, Odense University Hospital 5000 Odense, Denmark, Tel 654I 1350,Email anette.kjeldsen@rsyd.dk

Received: April 14, 2015 | Published: June II, 2015

\section{Introduction}

Hereditary Hemorrhagic Telangiectasia (HHT), also known as Osler-Weber-Rendu disease, is an autosomal dominant disorder. Genetic heterogeneity has been demonstrated with identification of five loci to date. The most common are HHT1 and HHT2. The reported prevalence of HHT in Denmark is around $1 / 6500 .{ }^{1}$ The disease is clinically characterized by a wide variety of clinical manifestations due to the presence of multiple Arterio venous malformations (AVMs). ${ }^{1}$ In some patients symptoms are subtle and result in minor inconvenience only, although the severity of symptoms normally increases with age. ${ }^{1,2}$ The most common clinical manifestation is spontaneous and recurrent epistaxis, usually beginning in childhood, ultimately affecting around $95 \%$ of all HHT patients. Other common clinical manifestations include: Gastrointestinal AVM (GI-AVM) Pulmonary arterio venous malformations (PAVMs), Cerebral AVM and Hepatic AVM. ${ }^{3,4}$ Although laryngeal bleeding is well described as a cause of hoarseness, ${ }^{5}$ laryngeal telangiectatic lesions causing hoarseness in an HHT patient has not previously been described. However recently a case report concerning an HHT patient with vocal fold scarring due to vocal fold haemorrhage was published. ${ }^{6}$ Treatment of HHT is mainly symptomatic, including removal of telangiectatic lesions using f.ex laser. Medical treatment with anti angiogenic drugs has been suggested but still needs further evaluation.

Acoustic voice analysis encompass objective assessment of 1) voice capacity obtained by the phonetogram which in a twodimensional histogram displays the sound intensity range measured in $\mathrm{dB}$ SPL over the range of fundamental frequencies (F0) of a given voice $^{7}$ and 2 ) voice quality, which encompass specific phonatory and resonator characteristics of the individual voice.

Acoustic voice analysis can be employed to both healthy and pathological voices and is widely used for monitoring effects of different types of treatments to the voice. ${ }^{8,9}$

\section{Case report}

The case concerns a 69 year old female patient, previously diagnosed with HHT. The patient was diagnosed with HHT in 1984.
Mutation analysis in the family revealed that the patient has HHT1, having a mutation in ENG: c. $360 \mathrm{C}>\mathrm{A}$, a well described mutation affecting several Danish families. ${ }^{10}$ The patient had PAVM which were treated by surgery and embolization in 1993, 1998 and again in 2012.

In February 2013 she contacted our department because of hoarseness during almost six months, and now she was only able to whisper. Furthermore she had experienced bleeding when coughing without relation to nosebleed, and had a clear sensation that the blood came from the larynx. Her private ENT doctor had visualised a red spot on her vocal cord and advised examination at our University clinic.

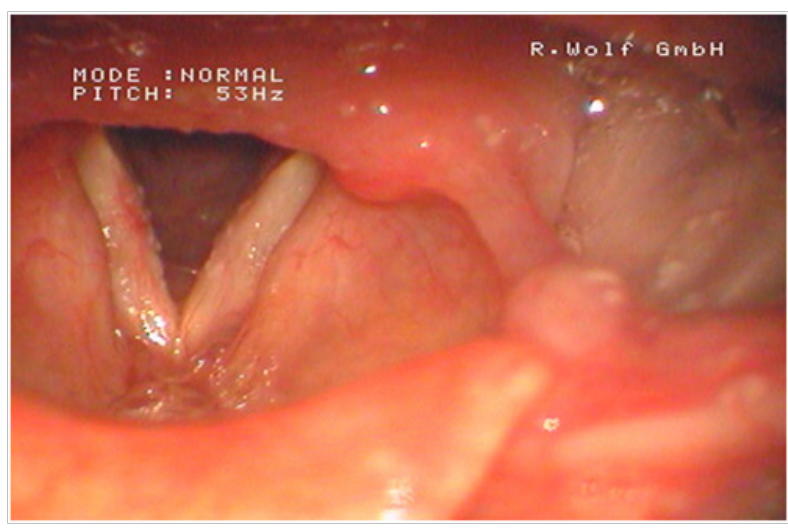

Figure I Stroboscopy showing telangiectatic lesion on the right vocal fold.

The patient went through thorough evaluation in our voicedisorder clinic, by that time she was almost aphonic. Video laryngostroboscopy (Figure 1) showed a relatively large telangiectatic lesion at the right vocal cord, the lesion was surrounded by hyperaemia and we found minor impaired vibration of the mucosa. Furthermore very small whitish spots on both vocal folds were noted. Pre-operative blood tests showed that the haemoglobin level was $5,3 \mathrm{mmol} / \mathrm{L}$, (7, $3-9,5 \mathrm{mmol} / \mathrm{L})$. Anaemia was primarily caused by gastrointestinal bleeding, as the frequency and grade of epistaxis was moderate at the time of investigation (epistaxis once a week). 
Microlaryngoscopy in general anesthesia was performed and the laryngeal telangiectatic lesion was treated by laser surgery, using the Biometic Laser 910nm, continuous mode, 9watt.

A laryngeal swap was performed and antifungal therapy prescribed (Fluconazol $100 \mathrm{mg}$ per day in 7 days). The culture was however without fungal growth.

The voice improved markedly after only a few weeks. At followup video laryngo-stroboscopy Figure 2 showed a completely normal larynx without any signs of telangiectatic lesions. At the follow-up an overall improvement in the voice capacity was apparent (Figure $3 \& 4$ ). The frequency range was 7 semitones higher (improvement in both lowest and highest frequencies). The intensity range went from $46 \mathrm{~dB}$ SPL to $66 \mathrm{~dB}$ SPL, an improvement of $20 \mathrm{~dB}$ SPL (11 $\mathrm{dB}$ SPL in maximum intensity and $9 \mathrm{~dB}$ SPL in minimum intensity). This means that the patient was able to sustain phonation using lower subglottic pressure after the surgery due to a better vocal fold closure. Also higher subglottic pressure could now be generated for maximum intensities. On the contrary, MDVP analysis displayed no improvement in voice quality.

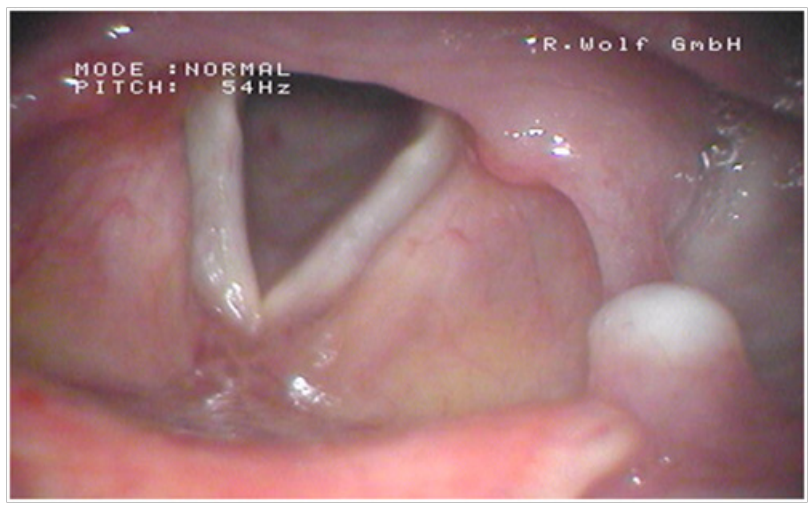

Figure 2 Stroboscopy 6 months after laser treatment of the right vocal fold.

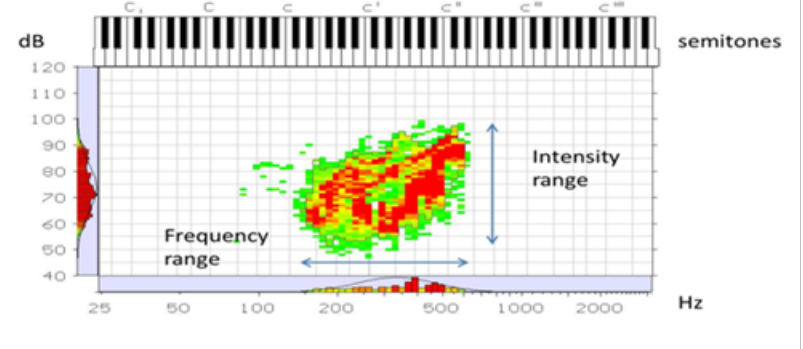

Figure 3 Phonetogram showing the patient's voice capacity before treatment.

Intensity range (Highest intensity - Softest intensity) marked by the arrow is $46 \mathrm{~dB}(97,5 \mathrm{~dB}-51,5 \mathrm{~dB})$ Frequency range (Number of semitones between Highest frequency and Lowest frequency) marked by the arrow is 25 semitones.

The voice improved due to better closure of the vocal fold vibration and the pressure needed to make the vocal folds vibrate decreases. The patient is able to speak with higher intensity with less effort than previously.

\section{Discussion and conclusion}

Hereditary Haemorragic Telangiectasia is a vascular disease involving the postcapillary venule causing dilatation of the capillary bed. Small capillary lesions $1-3 \mathrm{~mm}$ are very common in the nasal mucosa, on the lips and the tongue, and may be distributed throughout the gastrointestinal tract. Although it has not previously been described in the literature it comes as no surprise that these lesions also may occur on the vocal cord. The mechanism for development of telangiectatic lesions in HHT is hypothesized to be repetitive trauma of the mucosa. In the nose high turbulent airflow seems to be part of the explanation.

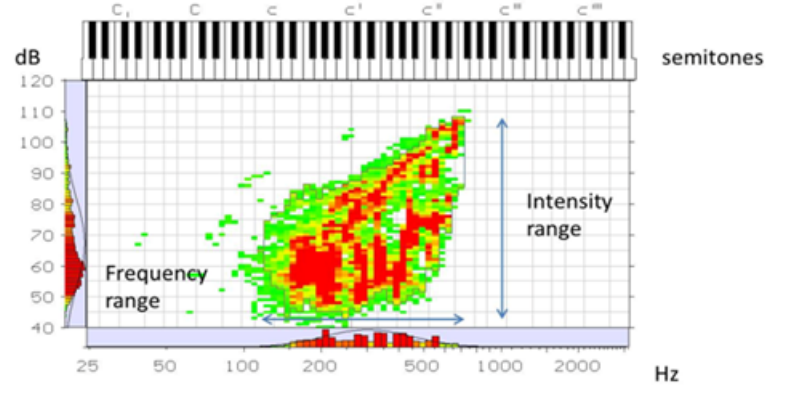

Figure 4 Phonetogram 6 months post laser treatment showing increased frequency and intensity ranges Intensity range (arrow) is $66 \mathrm{~dB}(108,5 \mathrm{~dB}-42,5$ $\mathrm{dB}$ ) Frequency range (arrow) is 32 semitones.

The vocal cords are also situated near high airflow, and still telangiectatic lesions in the vocal cords seem to be very rare. Our patient experienced severe voice problems. She had been treated with inhaled steroids because of asthma for some years. Laryngeal candidias is a well known side effect to inhaled steroids and cause of hoarseness. ${ }^{11}$ However stroboscopy showed an inflammatory reaction of both vocal folds as well as a telangiectatic lesion and hyperaemia only of the right fold. The inflammatory reaction could very well be a mechanical reaction due to an acute bleeding of the telangiectatic lesion. ${ }^{5}$ Although candida infection may also have been present, this could not be confirmed by relevant tests in our patient, and it was not considered to be the reason of the severe dysphonia our patient experienced. We therefore conclude that the telangiectatic lesion on our patient's vocal cord was the main reason for her voice problems.

Patients with HHT who complain of hoarseness should be referred to an otolaryngologist for evaluation. If telangiectatic lesions are identified at the vocal cords, these may explain the hoarseness and should be treated with laser surgery, in order to improve the voice and prevent re-bleeding. ${ }^{5}$ The treatment can be performed without any harm to the vocal mucosa, and a marked improvement of the voice may be noted.

\section{Acknowledgments}

None.

\section{Conflicts of interest}

Author declares there are no conflicts of interest.

\section{Funding}

None.

\section{References}

1. Kjeldsen AD, Vase P, Green A. Hereditary haemorrhagic telangiectasia: a population-based study of prevalence and mortality in Danish patients. J Intern Med. 1999;245(1):31-39. 
2. Plauchu H, de Chadarevian JP, Bideau A, et al.Age-related clinical profile of hereditary hemorrhagic telangiectasia in an epidemiologically recruited population. Am J Med Genet. 1989;32(3):291-297.

3. Kjeldsen $\mathrm{AD}$, Kjeldsen J. Gastrointestinal bleeding in patients with hereditary hemorrhagic telangiectasia. Am $J$ Gastroenterol. 2000;95(2):415-418.

4. Faughnan ME, Palda VA, Garcia-Tsao G, et al. International guidelines for the diagnosis and management of hereditary haemorrhagic telangiectasia. J Med Genet. 2011;48(2):73-87.

5. Lennon CJ, Murry T, Sulica L. Vocal fold hemorrhage: factors predicting recurrence. Laryngoscope. 2014;124(1):227-232.

6. Chang J, Yung KC. Dysphonia and vocal fold telangiectasia in hereditary hemorrhagic telangiectasia. Ann Otol Rhinol laryngol. 2014;123(11):769-770.
7. Lamesch S, Doval B, Castellengo M. Toward a more informative voice range profile: the role of laryngeal vibratory mechanisms on vowels dynamic range. $J$ Voice. 2012;26(5):672 e9-e18.

8. Speyer R, Wieneke GH, van Wijck-Warnaar I, et al.Effects of voice therapy on the voice range profiles of dysphonic patients. $J$ Voice. 2003;17(4):544-556.

9. Friedrich G, Dejonckere PH. [The voice evaluation protocol of the European Laryngological Society (ELS) -- first results of a multicenter study] Laryngorhinootologie. 2005;84(10):744-752.

10. Torring PM, Brusgaard K, Ousager LB, et al.National mutation study among Danish patients with hereditary haemorrhagic telangiectasia. Clin Genet. 2014;86(2):123-133.

11. Wong KK, Pace-Asciak P, Wu B, et al.Laryngeal candidiasis in the outpatient setting. J Otolaryngol Head Neck Surg. 2009;38(6):624-627. 